\title{
Spreading depolarization, Tsunami im Hirn
}

\author{
Jens P. Dreier
}

\begin{abstract}
Zusammenfassung
Spreading depolarization (SD) beschreibt eine neuronale und astrozytäre Massendepolarisationswelle der grauen Substanz. Normalerweise ist an diese eine Vasodilatation gekoppelt, die zu einem Blutflussanstieg führt (spreading hyperemia). Unter bestimmten Bedingungen kann SD aber auch einen arteriellen Spasmus der Mikrozirkulation mit konsekutiver spreading ischemia (SI) induzieren. Diese inverse neurovaskuläre Kopplung tritt z.B. unter artifizieller zerebrospinaler Flüssigkeit auf, die den Zustand nach Subarachnoidalblutung imitiert. SD mit normaler neurovaskulärer Kopplung induziert in gesundem Hirngewebe keinen Zelltod, während SI bei der Ratte kortikale Nekrosen verursacht. Im menschlichen Hirn sind kortikale Nekrosen typisches Korrelat verzögerter ischämischer Schlaganfälle nach Subarachnoidalblutung. Basierend auf unseren tierexperimentellen Befunden stellten wir 1998 die Hypothese auf, dass SI ein Mechanismus verzögerter ischämischer Schlaganfälle beim Menschen sein könnte. Mithilfe subduraler Opto-/Elektrodenstreifen und serieller Bildgebung konnten wir mittlerweile nicht nur das massive Auftreten von SDs nach Subarachnoidalblutung beim Menschen nachweisen, sondern auch das Auftreten von SI in der Entstehung verzögerter ischämischer Schlaganfälle. SI ist ein vielversprechendes Ziel für die Entwicklung neuer Therapiestrategien.
\end{abstract}

\begin{abstract}
Spreading depolarization, Tsunami in the brain.

The term spreading depolarization (SD) describes a wave of mass neuronal/astroglial depolarization in grey matter. Under normal conditions, spreading vasodilation is coupled to SD leading to spreading hyperemia. In contrast, under pathological conditions, SD induces severe microvascular spasm leading to spreading ischemia (SI). This inverse neurovascular coupling occurs, e.g., under artificial cerebrospinal fluid of composition designed to replicate that present following subarachnoid hemorrhage. Whereas SD with normal coupling does not induce neuronal death in healthy tissue in rats, SI leads to widespread cortical necrosis. In human brain, cortical necrosis is typically associated with delayed ischemic stroke, the most important in hospital complication of subarachnoid hemorrhage. In 1998 we hypothesized that SI is a mechanism of delayed ischemic stroke. Using opto-/electrode strips and serial neuroimaging, we have not only shown recently the abundant occurrence of SDs in patients with subarachnoid hemorrhage but also the occurrence of SI during the evolution of delayed ischemic stroke. SI is a promising target for treatment development.
\end{abstract}

Keywords: subarachnoid hemorrhage; ischemia; spreading depression; spreading ischemia

\section{Einleitung}

Der Begriff spreading depolarization (SD) beschreibt eine fast vollständige neuronale Massendepolarisation, die mit einer Nervenzellschwellung assoziiert ist und als Welle mit etwa $3 \mathrm{~mm} / \mathrm{min}$ in der grauen Substanz wandert. SD entsteht, wenn der positive Kationeneinstrom über die Zellmembran den Ausstrom über ATPabhängige Natrium- und Kalziumpumpen übersteigt. Der Kationeneinstrom wird von einem Wassereinstrom und einer Schrumpfung des Extrazellulärvolumens um $70 \%$ gefolgt (Somjen 2004). Wenn die normale Ionenhomöostase nicht durch zusätzliche Rekrutierung von Natrium- und Kalziumpumpenaktivität wiederhergestellt wird, bleibt die Zellschwellung bestehen. Dieser Prozess wird dann als ,zytotoxisches Ödem' bezeichnet, weil er u.a. durch die verlängerte zelluläre Kalziumüberladung und mitochondriale Depolarisation potenziell zum Zelltod führt.
SD führt zu einem Depolarisationsblock der Aktionspotenzialgenerierung, der eine spreading depression der elektrokortikografischen Aktivität nach sich zieht, wenn die elektrokortikografische Aktivität nicht bereits durch sedierende Medikamente, vorhergehende SDs oder einen schweren Energiemangel aufgehoben ist (Abbildung 1).

Die spreading depression ist somit eine Eigenschaft der SD und stellt nicht ihren Kernprozess dar. Der Kernprozess ist die nahezu vollständige Nervenzelldepolarisation (Somjen 2004).

Die spreading depression der elektrokortikografischen Aktivität wurde durch den brasilianischen Physiologen Aristides Leão im Kaninchenkortex 1944 entdeckt (Leão 1944). Drei Jahre später beschrieb er auch die große negative Gleichstrompotenzialveränderung (engl.: direct current (DC)) des Extrazellulärraums, die die Nervenzelldepolarisation begleitet. Darüber hinaus zeigte Leão, dass eine ähnliche wandernde DC - Negativierung im Kortex auch als Antwort auf einen beidseitigen Verschluss der A. carotis interna auftritt und folgerte aus seinen Experimenten, dass "in der spreading depression der [elektrokortikografischen] Aktivität eine Veränderung auftritt, die die gleiche Natur hat wie die, die nach einer länger dauernden Unterbrechung des systemischen Kreislaufs im Kortex beobachtet wird“ (Leão 1947). Vermutlich sind Spannungsinhomogenitäten entlang der neuronalen Zellmembranen für die große negative DC - Änderung während SD verantwortlich (Canals et al. 2005).

Leão und Morison (1945) stellten auch die Hypothese auf, dass SD das pathophysiologische Korrelat der Migräneaura ist. Somit hat Leão bereits in den Vierziger Jahren des letzten Jahrhunderts in den drei genannten bahnbrechenden Arbeiten im Journal of Neurophysiology die gesamte Bandbreite der SD - Erkrankungen erkannt, die von der relativ harmlosen Migräneaura bis zur Nervenzellreaktion bei Sauerstoff- oder Glukosemangel und allen Formen des Schlaganfalls reicht. Bei letzteren ist SD der Prozess, der den Nervenzelltod einleitet.

Obwohl SD mit der bei weitem größten Potenzialänderung assoziiert ist, die im Extrazellulärraum des Hirns auftreten kann und diese mehr als fünfmal größer ist als die Potenzialänderungen während epileptiformer Aktivität, lässt sie sich bisher nicht im Oberflächen-EEG des Menschen nachweisen. Das erklärt sich daraus, dass die Hirnhäute und der Schädel einen sehr großen kapazitiven Widerstand darstellen, der die im Sekundenbereich auftretenden und damit sehr langsamen Potenzialänderungen der SD wegfiltert. Die SD assoziierte Depression der 
schnellen Frequenzkomponenten ist ebenfalls nicht auf triviale Weise zu messen, da sich die Aktivität im Oberflächen-EEG aus einem sehr großen Kortexareal mit mehreren Quadratzentimetern Ausdehnung speist. Durch Überlagerung noch vorhandener Aktivität in angrenzenden Kortexarealen wird die lokale Depression elektrokortikografischer Aktivität somit einfach überdeckt.

Die fehlende Messbarkeit der SD im Oberflächen-EEG hat dazu geführt, dass Leãos Entdeckung über sechs Jahrzehnte keine Bedeutung für die Neurologie erlangte, obwohl die jetzt vorliegenden invasiven Messergebnisse beim Menschen Leãos Vermutungen bestätigen, dass SD das mit weitem Abstand wichtigste pathophysiologische Phänomen des Hirns ist, welches in mehr als $50 \%$ der Fälle für die neurologischen Defizite in der Notfallmedizin verantwortlich ist, d.h. bei allen Patienten mit Störungen des zerebralen Energiestoffwechsels angefangen vom Schlaganfall bis zu den neurologischen Defiziten nach einem Herzstillstand. Ziel der vorliegenden Arbeit ist es, einen Einblick in die fundamentale Rolle von SD beim Schlaganfall zu geben. Ein Schwerpunkt liegt auf der Störung der neurovaskulären Kopplung an SD.

\section{SD bei fokaler Ischämie}

Die fokale zerebrale Ischämie unterscheidet sich von der globalen Ischämie dadurch, dass sie Gradienten von Perfusion, Sauerstoff und Glukose aufweist, die vom Ischämiekern zum gesunden umgebenden Gewebe hin ansteigen. Aus Studien mit Mikroelektroden und optischen Verfahren existiert experimentelle Evidenz, dass eine lang dauernde, pharmakoresistente und den Zelltod initiierende SD im Zentrum des Niedrig-Perfusionsareals beginnt und sich in ihrer Wanderung durch die Ischämierandzone, die sogenannte Penumbra, und weiter in das gesunde Gewebe hinein in eine kurz dauernde, pharmakosensible und harmlose SD verwandelt (Busch et al. 1996; Petzold et al. 2005; Shin et al. 2006; Strong et al. 2007). Wichtig ist, dass in den verschiedenen Zonen nicht unterschiedliche Wellen entstehen, sondern es sich um jeweils eine Welle handelt, die ihren Charakter während ihrer Wanderung durch die verschiedenen Zonen abhängig vom lokalen Energiestatus verändert. Die Wellen entstehen repetitiv und mit jeder Welle wächst der nekrotische Kernbereich (Mies et al. 1993). Während SD in gesundem Gewebe durch NMDARezeptorantagonisten blockierbar ist, legen Untersuchungen von Müller und Somjen (1998) nahe, dass sich die Pharmakoresistenz der SD gegenüber NMDA - Rezeptorantagonisten im Kernbereich des Energiemangels durch eine zusätzliche Rekrutierung weiterer Kationenkanäle in den SD - Prozess erklärt. Die progressive Pharmakoresistenz von SD unter Energiemangelbedingungen hat vermutlich eine kritische Rolle beim Versagen der klinischen NMDA-Rezeptorantagonisten-Studien beim Schlaganfall gespielt.

Besonders deutlich lässt sich die Rolle von SD im Endothelin1-Modell der fokalen zerebralen Ischämie bei der Ratte darstellen. Endothelin-1 ist der derzeit potenteste Vasokonstriktor in der zerebralen Zirkulation. Hirntopische Applikation von Endothelin-1 erlaubt es, eine fokale zerebrale Ischämie konzentrationsabhängig und selektiv in einer kleinen kortikalen Zone zu erzeugen, die über ein kranielles Fenster zugänglich ist. Die Messung der SDs erfolgt hierbei mit Oberflächen- oder Tiefenelektroden. Unter Halothan-Anästhesie entwickelten ca. 50 \% der Tiere SD bei einer Endothelin-1- Konzentration von $1 \mu \mathrm{M}$, während SD in den übrigen $50 \%$ ausblieb. Bei diesen Experimenten zeigte sich, dass stets ein kleines kortikales Areal im Fensterbereich eine selektive neuronale Nekrose aufwies, wenn Endothelin-1 SD induzierte, nicht aber, wenn keine SD auftrat. Darüber hinaus fand sich ein ähnliches Schadensareal wie nach Endothelin-1-induzierter SD, wenn eine SD außerhalb des Fensters durch Kaliumchlorid induziert wurde und von außen in die Endothelin-1-exponierte Fensterregion hineinwanderte. Wenn keine SD durch Endothelin-1 induziert wurde und auch keine SD von außen durch den Endothelin-1-exponierten Kortex wanderte, entwickelte sich auch kein neuronaler Schaden. Diese Experimente zeigen, dass SD notwendig ist, um die Schadenskaskaden zum Zelltod in einem metabolisch kompromittierten Kortex zu initiieren, wobei es egal ist, ob die SD im metabolisch kompromittierten Bereich selbst entsteht oder von außen einwandert. Dagegen ist SD im gesunden Gewebe ein eher harmloser Prozess (Dreier et al. 2007).

\section{Physiologische neurovaskuläre Kopplung an SD}

Obwohl SD per se ein passiver Prozess ist, der durch Ladungs- und Ionenkonzentrationsgefälle über die Zellmembran getrieben wird, steigt der Energieverbrauch paradoxerweise an, da es unmittelbar zu einer Aktivierung von Natrium- und Kalziumpumpen kommt, um die intrazelluläre Natrium- und Kalziumüberladung zu beseitigen. So kommt es sogar in gesundem Gewebe zu einem Abfall der ATP - Gewebskonzentration um 50 \% (Mies und Paschen 1984). Um die Sauerstoff- und Glukoseverfügbarkeit zu steigern, induziert SD eine Dilatation der Widerstandsgefäße in gesundem Gewebe. Dadurch steigt der regionale

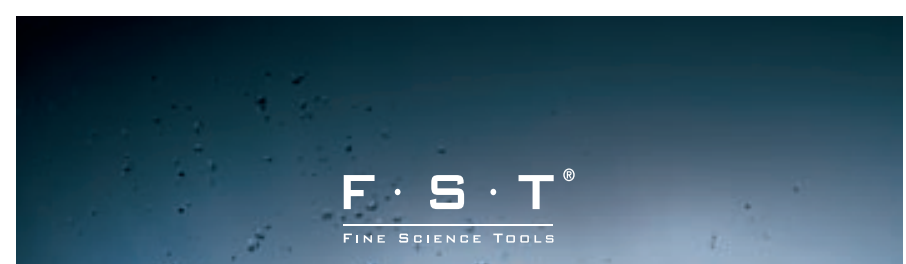

FINE SURGICAL INSTTRUMENTS FOR RESEARCHTM

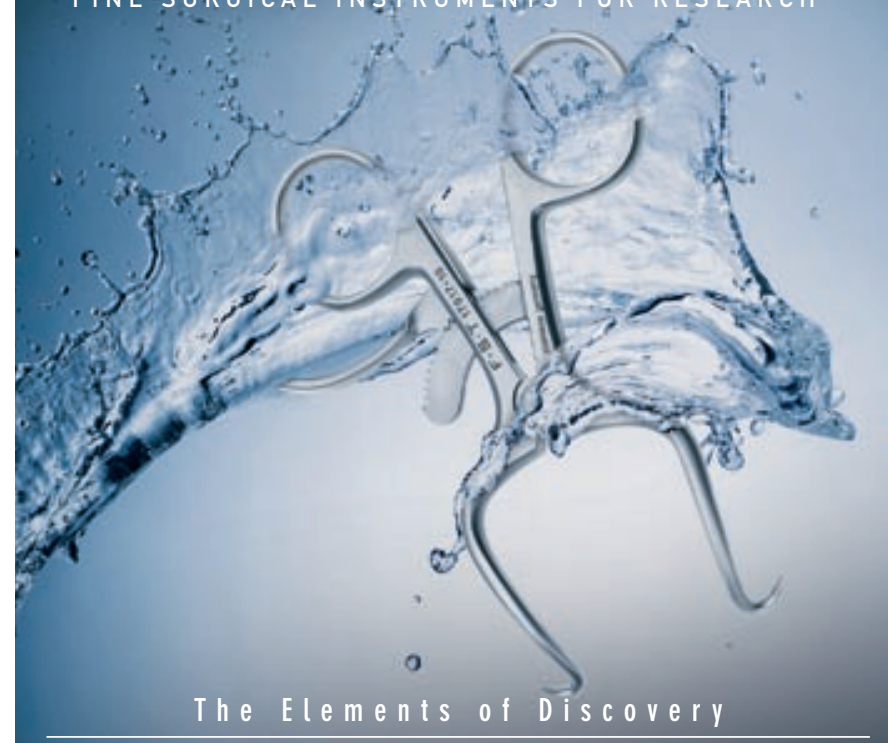

Scissors • Needle Holders • Forceps • Retractors • Clamps

Rongeurs • Probes • Scalpels • Surgical \& Laboratory Accessories Student Quality Instruments

Request a catalog at finescience.de or call +49(0) 6221905050 


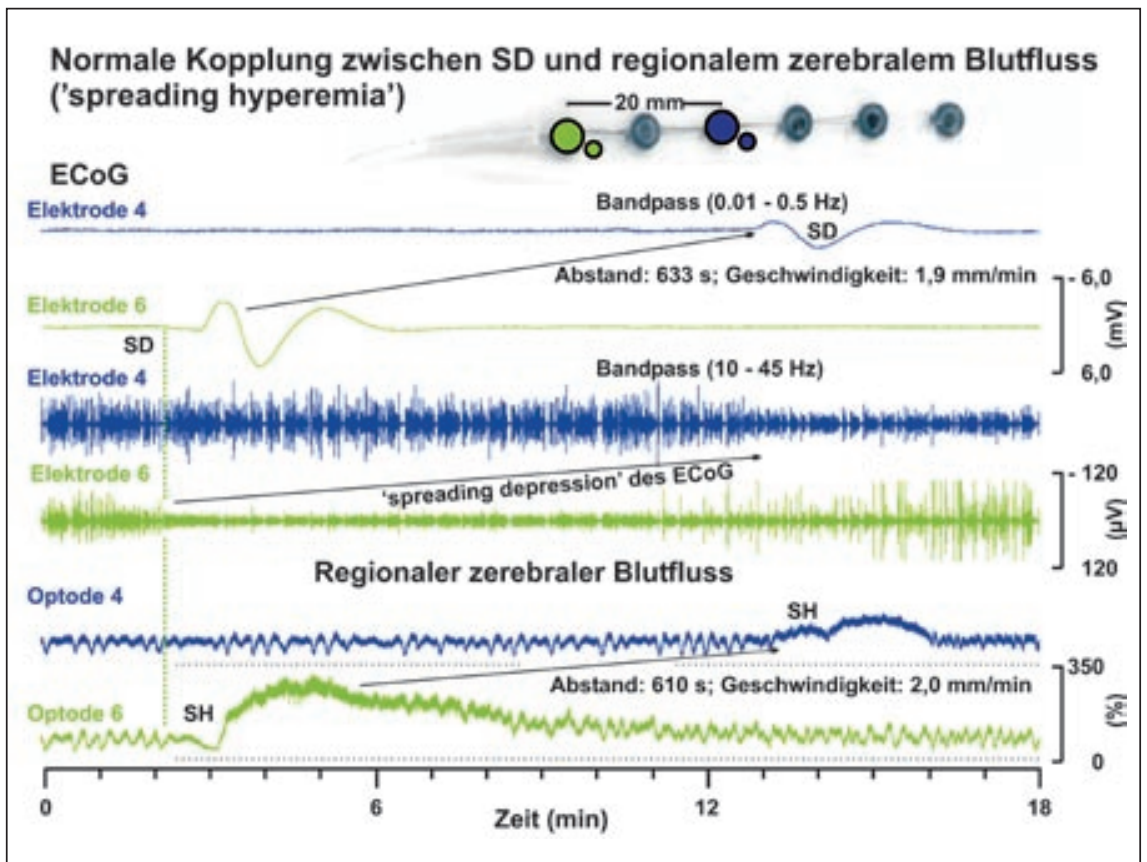

Abb. 1: Normale neurovaskuläre Kopplung an SD im menschlichen Hirn. Oben rechts ist der subdurale Opto-/Elektrodenstreifen gezeigt. Die sechs Spuren repräsentieren simultane Ableitungen einer einzelnen SD, die von Opto-/Elektrode 6 (blau) nach 4 (grün) wandert. Die Kalibrationsbalken gelten auch für die jeweils darüberliegende Spur. Die vier oberen Spuren identifizieren die SD elektrophysiologisch. Spur 1 und 2: bandpassgefiltertes Elektrokortikogramm (engl.: electrocorticogram (ECoG)) (Frequenzbereich: $0.01-0.5 \mathrm{~Hz}$ ), welches typische langsame Frequenzkomponenten einer SD zeigt (nicht zu verwechseln mit dem echten DC -Signal). Die SD breitet sich mit einer Geschwindigkeit von etwa $1,9 \mathrm{~mm} / \mathrm{min}$ aus, wenn man eine lineare Ausbreitung entlang des Elektrodenstreifens annimmt. Spur 3 und 4: bandpass-gefiltertes Elektrokortikogramm (10 - $45 \mathrm{~Hz})$, welches die typische spreading depression der schnellen Frequenzkomponenten zeigt. Spur 5 und 6: Typischer Blutflussanstieg während der Depolarisationsphase der SD (spreading hyperemia (SH)) bei normaler neurovaskulärer Kopplung gemessen mit Laser-DopplerFlussmessung.

zerebrale Blutfluss während der neuronalen Depolarisationsphase an (Abbildung 1). Erst nach der neuronalen Repolarisation folgt eine milde Vasokonstriktion auf die Phase der Gefäßdilatation, die zur sogenannten spreading oligemia führt (Lauritzen 1994).

Diese physiologische neurovaskuläre Kopplung während SD steht in scheinbarem Widerspruch zur klassischen Definition neurovaskulärer Kopplung, weil ein gesteigerter regionaler zerebraler Blutfluss an eine Abnahme der neuronalen Aktivität gekoppelt ist. Dieses Paradoxon lässt sich durch die Tatsache auflösen, dass SD zu einem Depolarisationsblock der Aktionspotenzialgenerierung führt. Die spreading depression der hochfrequenten elektrokortikografischen Aktivität reflektiert somit eine Form intensiver Exzitation im Gegensatz zu neuronaler Inhibition durch GABA-vermittelte Hyperpolarisation. Tatsächlich teilt SD eine Reihe von Eigenschaften mit physiologischer neuronaler Exzitation im Gegensatz zu neuronaler Inhi-
(Dreier et al. 1998; Shin et al. 2006). Das daraus resultierende wandernde Perfusionsdefizit (Abbildung 2) verlängert wiederum die neuronale Depolarisation und damit die Dauer der neuronalen Natrium- und Kalziumüberladung, weil der Sauerstoff- und Glukosemangel über eine Reduktion der ATP-Verfügbarkeit zu verminderter Aktivität der zellulären Natrium- und Kalziumpumpen führt (Dreier et al. 1998; Sukhotinsky et al. 2008). Dies zeigt sich extrazellulär in einer verlängerten negativen Verschiebung des kortikalen DC-Potenzials. Die Verlängerung des DC-Potenzials ist somit das elektrophysiologische Kriterium für die inverse neurovaskuläre Kopplung, die anzeigt, dass die SD-induzierte Hypoperfusion signifikant genug ist, um ein Missverhältnis zwischen neuronalem Energiebedarf und der Energieversorgung $\mathrm{zu}$ erzeugen. Entsprechend beschreibt der Begriff spreading ischaemia (SI) das mit der SD wandernde, initiale Perfusionsdefizit, wenn es zu einer verlängerten DC-Potenzialnegativierung im Kortex führt (Dreier et al. 1998).

Es konnte gezeigt werden, dass lang dauernde pharmakologisch induzierte SIs ausreichten, um weit ausgedehnte fokale Nekrosen der Hirnrinde bei der Ratte zu erzeugen, ohne dass vorher ein signifikantes Perfusionsdefizit z.B. durch proximalen Verschluss einer Hirnarterie bestanden hätte (Dreier et al. 2000). Diese Befunde legten nahe, dass die inverse neurovaskuläre Kopplung per se (i) ein Prozess ist, der die Wahrscheinlichkeit erhöht, dass SD Zellschaden induziert und sie daher (ii) ein vielversprechendes neues Ziel für therapeutische Interventionen darstellt (Dreier et al. 1998; 2000). Durch die inverse Kopplung wird eine vergleichsweise gutartige SD quasi in eine bösartige SD transformiert.

Das Phänomen der SI wurde in einem Rattenmodell für verzögerte ischämische Infarkte nach aneurysmatischer Subarachnoidalblutung entdeckt (Dreier et al. 1998). Dabei konnte gezeigt werden, dass SI auch rein pharmakologisch durch gleichzeitige Hemmung der NO - Synthase und Erhöhung der normalen Kaliumbasiskonzentration im Gewebe induziert werden kann (Dreier et al. 1998). Vasodilatatoren wie NO - Donoren oder L-Typ - Kalziumantagonisten waren in der Lage, die SI wieder in eine fast normale Blutflussantwort auf SD zurückzuverwandeln (Dreier et al. 1998; Windmüller et al. 2005). Später wurde SI auch in Rattenmodellen für systemische Hypotension (Sukhotinsky et al. 2008) und in der ischämischen Penumbra nach Verschluss der mittleren Zerebralarterie in Mäusen und Katzen nachgewiesen (Shin et al. 2006; Strong et al. 2007). 


\section{SD beim Menschen während Migräneaura}

Erste Messungen von SD beim Menschen wurden Ende der siebziger Jahre mit Tiefenelektroden im Nucleus caudatus und Hippocampus durchgeführt (Sramka et al. 1977/1978). In den achtziger Jahren wurden dann zerebrale Blutflussänderungen beim Menschen nuklearmedizinisch mit der planaren, intracarotidealen 133Xe-Methode sowie single-photon-emission computed tomography (SPECT) während Migräneaura nachgewiesen, die dem für SD aus Tierexperimenten bekannten Muster normaler neurovaskulärer Kopplung entsprachen (Olesen et al. 1981; Lauritzen 1994). Diese Befunde konnten mittels Positronenemissionstomografie bestätigt werden (Woods et al. 1994). 2001 gelang während Migräneaura beim Menschen mithilfe einer Hochfeldstärken-nah-kontinuierlichen MRT der Nachweis von acht SD-charakteristischen Änderungen im blood oxygen level-dependent (BOLD) -Signal einschließlich ähnlicher Dauer, Propagationsgeschwindigkeit und Depression lichtinduzierter neuronaler Aktivität (Hadjikhani et al. 2001). Weiterhin wurde SD zuverlässig in menschlichen Hirnschnitten elektrophysiologisch nachgewiesen, die nach epilepsiechirurgischen Eingriffen zur Verfügung standen (Avoli et al. 1991; Petzold et al. 2005).

\section{Nachweis von SD in Patienten mit akutem neuronalen Schaden}

Mayevsky und Mitarbeiter (1996) konnten SDs erstmals mittels einer invasiven Multiparametermessung, die u.a. DC-Potenzial, extrazelluläre Kaliumkonzentration und Blutfluss erfasste, bei einem von fünfzehn Patienten mit Schädel-Hirn-Trauma nachweisen. Strong und Mitarbeiter (2002) stellten dann ein methodisch einfaches Verfahren vor, das für ein Patientenmonitoring praktikabel ist und mit dem SDs in ungefähr $50 \%$ der Fälle bei Schädel-Hirn-Trauma-Patienten gemessen werden konnten. Dieses Verfahren beruht auf dem invasiven Nachweis der spreading depression hochfrequenter elektrokortikografischer Aktivität mittels eines subduralen Elektrodenstreifens. Ein solcher Elektrodenstreifen kann prinzipiell immer dann implantiert werden, wenn eine Indikation für einen neurochirurgischen Eingriff besteht. Nach der Akutphase kann der Streifen wieder herausgezogen werden, ohne dass eine erneute Operation erforderlich wäre. Gleichartige Elektrodenstreifen wurden schon seit Jahrzehnten in der prächirurgischen Epilepsiediagnostik eingesetzt. Lediglich die Verstärkereinstellungen unterscheiden sich hierbei.

Aus der Studie von Strong et al. (2002) entwickelte sich die Co-Operative Study on Brain Injury Depolarizations (COSBID), die zum Ziel hat, die Messung von SD für die Klinik nutzbar zu machen (www.cosbid.org). Fabricius et al. (2006) fanden im weiteren Verlauf auch langsamere Frequenzkomponenten in der subduralen Elektrokortikografie, mit denen SDs in Hirnregionen beim Menschen nachgewiesen werden konnten, die keine hochfrequente elektrokortikografische Aktivität mehr aufwiesen. Eine robuste Methode für eine subdurale echte DC-Potenzialmessung wurde schließlich von Dreier et al. (2009) eingeführt. Damit ist es nunmehr möglich, die Dauer der DC-Potenzialnegativierung als indirektes Maß für den Energiestatus des Gewebes zu bestimmen. Mittlerweile sind SDs nicht nur beim Schädel-HirnTrauma sondern auch bei spontanen intrazerebralen Blutungen (Fabricius et al. 2006), aneurysmatischer Subarachnoidalblutung (Dreier et al. 2006), verzögerten ischämischen Schlaganfällen nach Subarachnoidalblutung (Dreier et al. 2006) und malignen ischämischen Mediainfarkten (Dohmen et al. 2008) in einem hohen Prozentsatz der jeweiligen Patientenpopulationen ( 50-100\%) nachgewiesen worden. Insbesondere konnte mithilfe sequenzieller MRTs gezeigt werden, dass das Auftreten verzögerter ischämischer Schlaganfälle nach Subarachnoidalblutung in der Bildgebung mit dem plötzlichen Auftreten von Clustern wiederkehrender SDs mit stark verlängerten elektrokortikografischen Depressionsphasen assoziiert war (Dreier et al. 2006). Durch die Einführung von subduralen Opto-/Elektrodenstreifen zur kombinierten Messung von Elektrokortikogramm und regionalem zerebralen Blutfluss konnte schließlich auch das Auftreten von SIs in der Entstehung verzögerter ischämischer Schlaganfälle nachgewiesen werden (Dreier et al. 2009) (Abbildung 2).

\section{Ausblick}

In der modernen Neurobiologie geht es vielfach darum, immer kleinere und spezialisiertere Prozesse, immer besser aufzulösen. Das Problem mit der SD war in den vergangenen sechs Jahrzehnten aber stets ein anderes: Ihre Wellenfront ist zu flach, um trotz ihrer enormen Größe mit einfachen Mitteln im Oberflächen-EEG sichtbar zu sein und sie spielt eine zu vielfältige pathophysiologische Rolle, um in der Komplexität ihres Auftretens sowohl bei relativ harmlosen Zuständen als auch bei schwersten Erkrankungen als ein - wie Leão es ausdrückte - , seiner Natur nach gleicher Prozess' erkannt zu werden. Für die Medizin der Zukunft ist die SD jedoch nicht nur als Ziel für neue Therapiestrategien hochin-

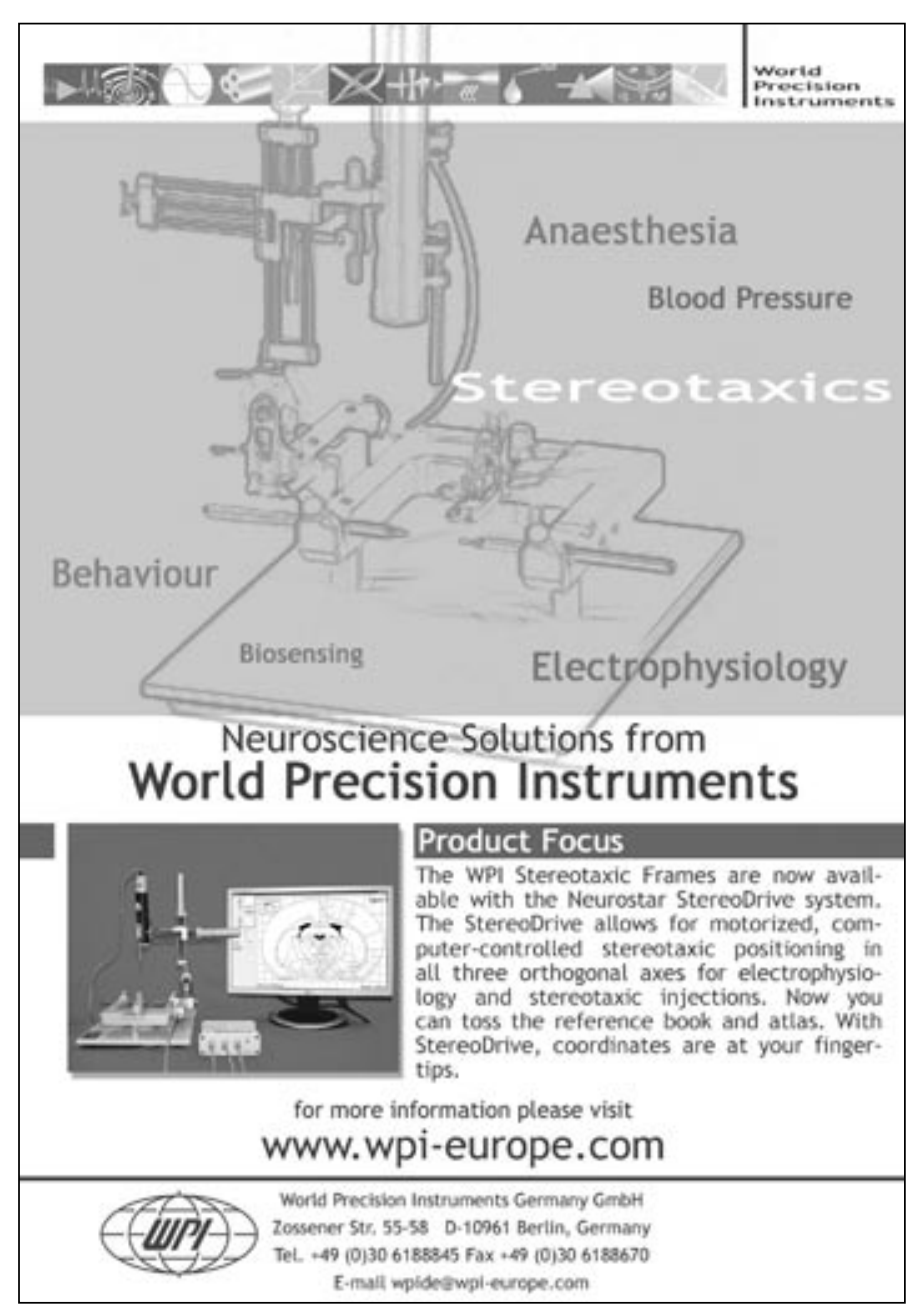




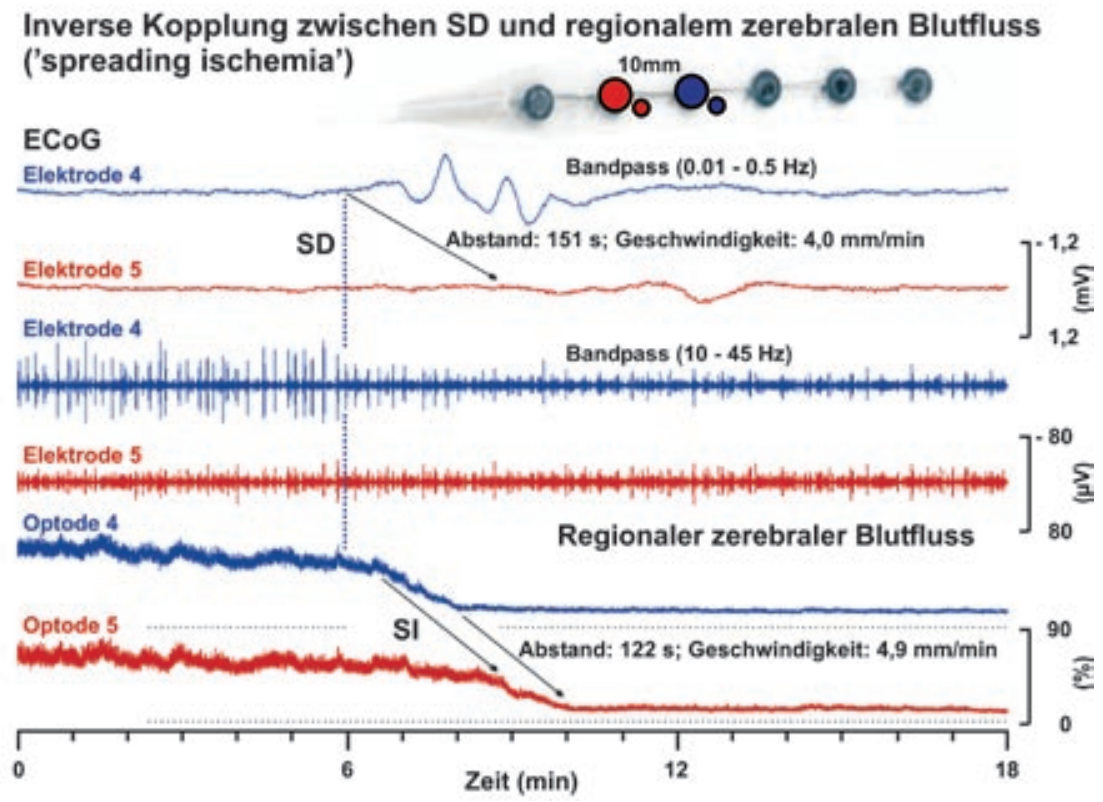

Abb. 2: Inverse neurovaskuläre Kopplung an SD im menschlischen Hirn nach aneurysmatischer Subarachnoidalblutung. Oben rechts ist der subdurale Opto-/Elektrodenstreifen gezeigt. Die sechs Spuren repräsentieren simultane Ableitungen einer einzelnen SD, die von Opto-/Elektrode 4 (blau) nach 5 (rot) wandert. Die Kalibrationsbalken gelten auch für die jeweils darüberliegende Spur. Die vier oberen Spuren identifizieren die SD elektrophysiologisch. Spur 1 und 2: bandpass-gefiltertes Elektrokortikogramm (engl.: electrocorticogram (ECoG)) $(0.01-0.5 \mathrm{~Hz})$ mit den typischen langsamen Frequenzkomponenten einer SD. Die SD breitet sich mit einer Geschwindigkeit von etwa $4,0 \mathrm{~mm} / \mathrm{min}$ aus. Spur 3 und 4: bandpass-gefiltertes Elektrokortikogramm (10 - $45 \mathrm{~Hz})$. Während die hochfrequente Aktivität in Elektrode 5 bereits durch eine vorhergehende SD supprimiert ist, kommt es in Elektrode 4 zu einer Depression der elektrokortikografischen Aktivität während der langsamen Potenzialverschiebung in Spur 1. Spur 5 und 6: Für die inverse neurovaskuläre Kopplung typischer Blutflussabfall während der kortikalen Depolarisationsphase (spreading ischemia (SI)). Beachte, dass die elektrophysiologischen Änderungen in Elektrode 4, den Blutflussänderungen in den Optoden vorausgehen und der Blutflussabfall von Optode 4 nach 5 wandert. Im zerebralen Computertomogramm wurde nach dem Ereignis ein Infarkt sichtbar, der das von den Elektroden erfasste Gebiet mitbetraf.

teressant sondern auch gerade deshalb, weil sie kein spezialisierter Prozess im Kleinen ist, sondern als Summenmaß für den drohenden Zelluntergang eines großen Nervenzellverbandes oder sogar des Gesamthirns gesehen werden kann. Anders als in der Biologie geht es in der Medizin ja in der Regel nicht darum, Einzelprozesse zu beschreiben, sondern aus der Messung komplexer Summenprozesse, wie z.B. der Messung eines EKGs, sinnvolle Entscheidungen für den gesamten kranken Menschen abzuleiten.

Aus Sicht der Medizin wäre es wünschenswert, wenn jeder bei Energiemangel beschriebene Schadens- oder Reparaturmechanismus in der grauen Substanz mittel- und langfristig in seiner Beziehung zum Summenphänomen SD eingeordnet werden würde. Da SD beim Menschen am Krankenbett jetzt messbar ist, wäre dadurch vermutlich eine verbesserte zeitliche Einschätzung möglich, in welcher klinischen Situation, welche Subprozesse von potenziellem Einfluss sind und welche Therapien entsprechend zukünftig wann zum Einsatz kommen könnten. Damit ist gemeint, dass die Aufzeichnung von SD es vermutlich nicht nur erlaubt, das Auftreten eines Schlaganfalls im Moment seiner Entstehung zu erfassen, sondern auch den Übergang der progressiven Schadensphase zur nachfolgenden Reparaturphase. Dieser findet vermutlich erst nach Tagen statt und dürfte vom Schweregrad des Schlaganfalls und dem Auftreten von Komplikationen abhängig und damit stark variabel sein (Dreier et al. 2009).

Ein solches Vorgehen könnte eine praktische Bedeutung für einen phasenspezifischen, d.h. zeitlich differenzierten Einsatz von Neuroprotektiva erlangen, da sich zunehmend herauskristallisiert, dass Signale wie z.B. die NMDA-Rezeptoraktivierung, die in der Akutphase zum Zelltod beitragen, in der Regenerationsphase an Prozessen der Reparatur und Plastizität beteiligt sind (Lo 2008). D.h., ob ein Medikament sich positiv oder negativ auf den Verlauf eines Schlaganfalls auswirkt, hängt davon ab, wann es gegeben wird.

Neben einem phasenspezifischen klinischen Einsatz von Medikamenten oder Interventionen zur Unterstützung von Reparatur und Regeneration könnte die Aufzeichnung von SDs auch für die Therapiesteuerung in der Akutphase von großem Wert sein. Dabei könnte es durch die Messung von SD als Summenmaß für den drohenden Zelluntergang in Zukunft möglich werden, eine frühe Therapiestratifikation durchzuführen. D.h. der Patient würde selektiv im Moment der Schadensentstehung einer bestimmten, möglicherweise nebenwirkungsreichen aber wirksamen Therapie zugeführt werden, während diese Therapie bei anderen Patienten, die keinen Schlaganfall entwickeln, vermieden wird. Dies könnte z.B. bei verzögerten ischämischen Schlaganfällen nach Subarachnoidalblutung eine interessante Option darstellen, da diese Form des Schlaganfalls erst Tage nach der eigentlichen Hirnblutung auftritt, während sich der Patient bereits auf der neurologischen Intensivstation befindet, wo das Auftreten von SDs online erfasst werden kann. Der Vorteil einer solchen Therapiestratifikation wäre, dass wirklich nur die Patienten behandelt würden, die von dem Medikament auch profitieren und bei denen man die potenziellen Nebenwirkungen daher in Kauf nehmen kann. Derzeit führen wir eine multizentrische prospektive klinische Studie durch, in der erstmals ein aussagekräftiger cutoff-Wert für die Dauer der SD-induzierten hochfrequenten elektrokoritikografischen Depression bestimmt werden soll, der das Auftreten eines verzögerten ischämischen Schlaganfalls beim Patienten anzeigt. Dieser cutoff -Wert soll dann später als Basis für eine Therapiestratifikation genutzt werden (DISCHARGE-1, DFG DR 323/5-1, ISRCTN05667702).

\section{Literatur}

Avoli, M., Drapeau, C., Louvel, J., Pumain, R., Olivier, A. und Villemure, J.G. (1991): Epileptiform activity induced by low extracellular magnesium in the human cortex maintained in vitro. Ann Neurol 30: 589-596.

Busch, E., Gyngell, M.L., Eis, M., Hoehn-Berlage, M. und Hossmann, K.A. (1996): Potassiuminduced cortical spreading depressions during focal cerebral ischemia in rats: contribution to lesion growth assessed by diffusion-weighted NMR and biochemical imaging. $J$ Cereb Blood Flow Metab 16: 1090-1099. 
Canals, S., Makarova, I., López-Aguado, L., Largo, C., Ibarz, J.M. und Herreras, O. (2005): Longitudinal depolarization gradients along the somatodendritic axis of CA1 pyramidal cells: a novel feature of spreading depression. J Neurophysiol. Aug 94 (2): 943-51.

Dohmen, C., Sakowitz, O.W., Fabricius, M., Bosche, B., Reithmeier, T., Ernestus, R.I. et al. (2008): Spreading depolarizations occur in human ischemic stroke with high incidence. Ann Neurol 63: 720-8.

Dreier, J.P., Körner, K., Ebert, N., Görner, A., Rubin, I., Back, T. et al. (1998): Nitric oxide scavenging by hemoglobin or nitric oxide synthase inhibition by $\mathrm{N}$-nitro-L-arginine induces cortical spreading ischemia when $\mathrm{K}^{+}$is increased in the subarachnoid space. J Cereb Blood Flow Metab 18: 978-90.

Dreier, J.P., Ebert, N., Priller, J., Megow, D., Lindauer, U., Klee, R. et al. (2000): Products of hemolysis in the subarachnoid space inducing spreading ischemia in the cortex and focal necrosis in rats: a model for delayed ischemic neurological deficits after subarachnoid hemorrhage? J. Neurosurg 93: 658-66.

Dreier, J.P., Woitzik, J., Fabricius, M., Bhatia, R., Major, S., Drenckhahn, C. et al. (2006): Delayed ischaemic neurological deficits after subarachnoid haemorrhage are associated with clusters of spreading depolarizations. Brain 129: 3224-37.

Dreier, J.P., Kleeberg, J., Alam, M., Major, S., KohlBareis, M., Petzold, G.C., Victorov, I., Dirnagl, U., Obrenovitch, T.P. und Priller, J. (2007): Endothelin-1-induced spreading depression in rats is associated with a micro area of selective neuronal necrosis. Exp Biol Med (Maywood) 232: 204-213 (IF 2.851).

Dreier, J.P., Major, S., Manning, A., Woitzik, J., Drenckhahn, C., Steinbrink, J., Tolias, C., Oliveira-Ferreira, A.I., Fabricius, M., Hartings, J.A., Vajkoczy, P., Lauritzen, M., Dirnagl, U., Bohner, G., Strong, A.J.; COSBID study group (2009): Cortical spreading ischaemia is a novel process involved in ischaemic damage in patients with aneurysmal subarachnoid haemorrhage. Brain. Jul;132(Pt 7): 1866-81.

Fabricius, M., Fuhr, S., Bhatia, R., Boutelle, M., Hashemi, P., Strong, A.J. et al. (2006): Cortical spreading depression and peri-infarct depolarization in acutely injured human cerebral cortex. Brain 129: 778-90.

Hadjikhani, N., Sanchez Del Rio, M., Wu, O., Schwartz, D., Bakker, D., Fischl, B., Kwong, K.K., Cutrer, F.M., Rosen, B.R., Tootell, R.B., Sorensen, A.G. und Moskowitz, M.A. (2001): Mechanisms of migraine aura revealed by functional MRI in human visual cortex. Proc Natl Acad Sci USA 10 98: 4687-4692.

Lauritzen, M. (1994): Pathophysiology of the migraine aura. The spreading depression theory. Brain 117: 199-210.

Leão (1944): Spreading depression of activity in the cerebral cortex. J Neurophysiol 7, 359-90.

Leão, A.A.P. (1947): Further observations on the spreading depression of activity in the cerebral cortex. J. Neurophysiol 10: 409-14.

Leão, A.A.P. (1945): Morison RS. Propagation of spreading cortical depression. J. Neurophysiol 8: 33-45.
Lo, E.H.A. (2008): New penumbra: transitioning from injury into repair after stroke. Nat Med 14: 497-500.

Mayevsky, A., Doron, A., Manor, T., Meilin, S., Zarchin, N. und Ouaknine, G.E. (1996): Cortical spreading depression recorded from the human brain using a multiparametric monitoring system. Brain Res. Nov 18;740(1-2): 268-74.

Mies, G. und Paschen, W. (1984): Regional changes of blood flow, glucose, and ATP content determined on brain sections during a single passage of spreading depression in rat brain cortex. Exp Neurol 84: 249-58.

Mies, G., Iijima, T. und Hossmann, K.A. (1993): Correlation between peri-infarct DC shifts and ischaemic neuronal damage in rat. Neuroreport 4: 709-711.

Müller, M. und Somjen, G.G. (1998): Inhibition of major cationic inward currents prevents spreading depression-like hypoxic depolarization in rat hippocampal tissue slices. Brain Res 812: 1-13.

Olesen, J., Larsen, B., Lauritzen, M. (1981): Focal hyperemia followed by spreading oligemia and impaired activation of rCBF in classic migraine. Ann Neurol. Apr;9 (4): 344-52.

Petzold, G.C., Windmüller, O., Haack, S., Major, S., Buchheim, K., Megow, D., Gabriel, S., Lehmann, T.-N., Drenckhahn, C., Peters, O., Meierkord, H., Heinemann, U., Dirnagl, U. und Dreier, J.P. (2005): Increased extracellular $\mathrm{K}^{+}$concentration reduces the efficacy of N-methyl-D-aspartate receptor antagonists to block spreading depression-like depolarizations and spreading ischemia. Stroke 36: 1270-1277.

Shin, H.K., Dunn, A.K., Jones, P.B., Boas, D.A., Moskowitz, M.A. und Ayata, C. (2006): Vasoconstrictive neurovascular coupling during focal ischemic depolarizations. $J$ Cereb Blood Flow Metab 26: 1018-30.

Somjen, G.G. (2004): Ions in the Brain. Normal Function, Seizures and Stroke. New York: Oxford University Press.

Sramka, M., Brozek, G., Bures, J., Nadvornik, P. (1977-78): Functional ablation by spreading depression: possible use in human stereotactic neurosurgery. Appl Neurophysiol 40: 48-61.

Strong, A.J., Fabricius, M., Boutelle, M.G., Hibbins, S.J., Hopwood, S.E., Jones, R., Parkin, M.C. und Lauritzen, M. (2002): Spreading and synchronous depressions of cortical activity in acutely injured human brain. Stroke Dec;33 (12): 2738-43.

Strong, A.J., Anderson, P.J., Watts, H.R., Virley, D.J., Lloyd, A., Irving, E.A. et al. (2007): Periinfarct depolarizations lead to loss of perfusion in ischaemic gyrencephalic cerebral cortex. Brain 130: 995-1008.

Sukhotinsky, I., Dilekoz, E., Moskowitz, M.A. und Ayata, C. (2008): Hypoxia and hypotension transform the blood flow response to cortical spreading depression from hyperemia into hypoperfusion in the rat. $J$ Cereb Blood Flow Metab 28: 1369-76.

Windmüller, O., Lindauer, U., Foddis, M., Einhäupl, K.M., Dirnagl, U., Heinemann, U. et al. (2005): Ion changes in spreading ischaemia induce rat middle cerebral artery constriction in the absence of NO. Brain 128: 2042-51.

Woods, R.P., Iacoboni, M. und Mazziotta, J.C. (1994): Bilateral spreading cerebral hypoperfu- sion during spontaneous migraine headache. $N$ Engl J Med 331: 1689-1692.

\section{Kurzbiografie}

Jens Dreier: geboren 1965; Studium der Medizin an der Freien Universität Berlin und der Universität zu Köln. Promotion 1993 in Köln. Habilitation in Neurologie 2003 an der Charité in Berlin. Seit 2006 Oberarzt der Neurologischen Klinik der Charité. Seit 2009 Professor für Translationale Schlaganfallforschung am Zentrum für Schlaganfallforschung (CSB) der Charité.

\section{Danksagung}

Die Arbeiten werden durch Mittel der Deutschen Forschungsgemeinschaft (DFG DR 323/3-1, 323/5-1, SFB Tr3 D10) sowie des Bundesministeriums für Bildung und Forschung (Center for Stroke Research Berlin 01 EO 0801), des Kompetenznetzes Schlaganfall und des Bernsteinzentrums II unterstützt.

\section{Korrespondenzadresse}

Prof. Dr. med. Jens P. Dreier

Center for Stroke Research Berlin

Charité-Universitätsmedizin Berlin

Charitéplatz 1, 10117 Berlin

Tel.: $\quad+4930450660024$

E-Mail: jens.dreier@charite.de

\section{Neueintritte}

Folgende Kolleginnen und Kollegen dürfen wir als Mitglieder der Neurowissenschaftlichen Gesellschaft begrüßen:

Ansorg, Anne (Jena)

Battefeld, Arne (Berlin)

Chaitidis, Dr. rer.nat Pavlos (Berlin)

Chauveau, Dr. Frédéric (Münster)

Gasser, Dr. rer.nat Andreas

(West Haven, USA)

Jansen, Sebastian (Berlin)

Jovalekic, Dipl-Biol. Aleksandar

(London, UK)

Kamin, Diplom Biologe Dirk (Göttingen)

Kröcher, Tim (Hannover)

Mutyala, Rithwik (Berlin)

Schanze, Prof. Dr. Thomas (Giessen)

Szukalski, Susann (Leipzig)

ten Bruggencate, Prof. em. Dr. med Gerrit (Berlin)

Der Mitgliedsstand zum 1. November 2009 beträgt 1.980 Mitglieder. 